\title{
Extrusión Apical de Barro Dentinario e Irrigante Produ- cidos por Dos Sistemas de Instrumentación de Níquel Titanio al Utilizar Irrigación Pasiva o Activa
}

\author{
Apical Extrusion of Dentin and Irrigant Smear Layer Produced by Two Nickel \\ Titanium Instrumentation Systems when Using Passive or Active Irrigation
}

\author{
Alfredo Sierra-Cristancho; Daniel Gómez Villarroel; Fabián Gajardo Martínez \& Verónica Correa Schnake
}

\begin{abstract}
SIERRA-CRISTANCHO, A.; GÓMEZ, V. D.; GAJARDO, M. F. \& CORREA, S. V. Extrusión apical de barro dentinario e irrigante producidos por dos sistemas de instrumentación de níquel titanio al utilizar irrigación pasiva o activa. Int. J. Odontostomat., 13(1):51-57, 2019.

RESUMEN: El barro dentinario producido durante tratamiento endodóntico puede ser extruido hacia el tejido periradicular junto con el irrigante, produciendo inflamación y dolor postoperatorio. Comparar la cantidad de extrusión apical de barro dentinario e irrigante, producido durante la preparación químico-mecánica, por dos sistemas de instrumentación Rotatoria (Mtwo), y Reciprocante (Reciproc), complementado con irrigación pasiva o activa (Endoactivator). Cuarenta y ocho premolares (48) inferiores, fueron aleatoriamente distribuidos en cuatro grupos de estudio ( $n=12$ dientes), (1) Rotatorio-pasiva, (2) Reciprocante-pasiva, (3) Rotatorio-activa y (4) Reciprocante-activa. Los conductos radiculares fueron instrumentados de acuerdo a las instrucciones del fabricante, e irrigados con hipoclorito de sodio al $5 \%$. El barro dentinario e irrigante extruido, fueron recolectados en tubos Eppendorf previamente pesados. Se calcularon los valores de extrusión de barro dentinario e irrigante para cada grupo. Los datos fueron analizados con el análisis de varianza ANOVA. En todos los grupos se produjo extrusión apical. No se encontraron diferencias estadísticamente significativas en la extrusión apical de barro dentinario, entre los grupos estudiados $(P=0,068)$, sin embargo, al analizar la extrusión de irrigantes, se evidenció diferencia estadísticamente significativa entre los grupos 1 y $4(P<0,05)$, entre los demás grupos no se observaron diferencias estadísticamente significativas. $(P>0,05)$. Bajo las condiciones de este estudio, los sistemas rotarios y reciprocante, extruyen sólido y líquido, siendo el sistema reciprocante el que produce mayor extrusión de líquido, tanto con irrigación activa como pasiva.
\end{abstract}

PALABRAS CLAVE: extrusión apical, barro dentinario, instrumentación mecanizada.

\section{INTRODUCCIÓN}

La Asociación Americana de Endodoncia, definió el barro dentinario, como una película de detritus retenido sobre la dentina u otra superficie, contiene partículas de dentina, remanentes de tejido pulpar vital o necrótico, bacterias e irrigantes. Producido durante la preparación químico mecánica (PQM) (American Association of Endodontists, 2015). Todas las técnicas e instrumentos endodónticos producen barro dentinario como consecuencia de su accionar sobre las paredes del conducto radicular (Violich \& Chandler, 2010), que puede ser extruido al tejido periradicular a pesar del estricto control de la longitud de trabajo. La extrusión de barro dentinario ha sido relacionado por algunos autores con reacciones inflamatorias, dolor post instrumen- tación, reagudizaciones y dificultad en la reparación de patologías apicales (Caviedes-Bucheli et al., 2013; Sipaviciute' \& Maneliene, 2014; Onay et al., 2015). Varios factores han sido relacionados con este fenómeno como la técnica de instrumentación, el tipo y volumen de la solución irrigante (Parirokh et al., 2012), características y profundidad de la aguja (Aksel et al., 2014; Chang, et al., 2015; Silva et al., 2016a,b), el uso de dispositivos de agitación de irrigantes como, ultrasonido, Endoactivator (Dentsply Maillefer, Ballaigues, Suiza), Endovac (Discus Dental, Culver City, USA), o láser(Alkahtani et et al., 2014; Gupta et al., 2014; Arslan et al., 2015; Helvacıoglu Kıvanç et al., 2015) y diámetro de la preparación apical (Teixeira et al., 2015). 
SIERRA-CRISTANCHO, A.; GÓMEZ, V. D.; GAJARDO, M. F. \& CORREA, S. V. Extrusión apical de barro dentinario e irrigante producidos por dos sistemas de instrumentación de níquel titanio al utilizar irrigación pasiva o activa. Int. J. Odontostomat., 13(1):51-57, 2019.

Se ha reportado mayor extrusión al utilizar técnicas manuales convencionales que al utilizar sistemas de níquel titanio (NiTi) accionados por motor (Ferraz et al., 2001; Sowmya et al., 2014). Recientemente se ha demostrado que el uso de movimiento recíproco aumenta la durabilidad de los instrumentos rotatorios NiTi e incrementa su resistencia a la fatiga en comparación al movimiento continuo. Con estas características han surgido los sistemas rotatorios de lima única de movimiento reciprocante, que permiten, en la mayoría de los casos, preparar el conducto radicular con un solo instrumento, lo que reduce el tiempo de trabajo por parte del operador (Caviedes-Bucheli et al., 2016), así como también el tiempo de acción de los irrigantes en el canal radicular, por lo que se hace necesario potenciar sus propiedades por medio del uso de dispositivos que activen o agiten los irrigantes, lo que podría aumentar el grado de extrusión de desechos a través del foramen (Yost et al., 2015; Huiz Peeters et al., 2018). El Endoactivator es un dispositivo de irrigación sónica, que opera en frecuencias que van desde 1-6 kHz. Consiste en una pieza de mano portátil y tres tipos de puntas de polímero flexible que no cortan la dentina, su diseño permite la activación y agitación de sustancias irrigantes intracanal (Rodríguez-Figueroa et al., 2014). Los sistemas reciprocantes de lima única producen mayor cantidad de extrusión que los sistemas rotatorios de múltiples limas (Kustarci et al., 2008; Bürklein \& Schäfer, 2012; Nayak et al., 2014; Nevares et al., 2015; Lu et al., 2015; Caviedes-Bucheli et al., 2016; Silva et al., 2016a,b).

El objetivo de este estudio es hacer un análisis comparativo in vitro, cuantificando la extrusión apical de barro dentinario (sólido) e irrigante (líquido), producida por los sistemas de instrumentación rotatoria continua Mtwo y reciprocante Reciproc, complementado con el uso de irrigación pasiva y activa (Endoactivator) durante la PQM del conducto radicular.

\section{MATERIAL Y MÉTODO}

Selección de muestras: Se seleccionaron cuarenta y ocho (48) premolares inferiores extraídos por razones ortodónticas o periodontales, todos los procedimientos se realizaron de acuerdo con las disposiciones éticas y legales vigentes. Los dientes fueron radiografiados en sentido vestíbulo-lingual y mesio-distal, para evidenciar la presencia de un único conducto radicular, con una raíz recta y ausencia de calcificaciones, de reabsorciones internas o tratamientos endodónticos previos.
Preparación de muestras: Las raíces fueron limpiadas y alisadas con curetas periodontales y discos Sofflex (3M) en motor de baja velocidad, a las cuales se les aplicaron tres capas de barniz (Kus, tarci et al.), con la finalidad de evitar microfiltraciones.

Diseño de cavidad de acceso: Se utilizó una fresa redonda de diamante $N^{\circ} 8$ (SS White, USA) y fresa EndoZ (Dentsply Maillefer) en turbina de alta velocidad refrigerada, los remanentes de tejido pulpar fueron extirpados con un extractor pulpar, con la precaución de no pasar más allá' del tercio medio con el instrumento.

Exploración del conducto: Con una lima $\mathrm{K} \mathrm{N}^{\circ} 15$ se exploró el conducto radicular en toda su extensión hasta alcanzar el foramen apical, constatándose a través de un examen visual con ayuda de una lupa de $4 X$. El tope de silicona se ajustó a la cúspide vestibular. El instrumento fue removido y con ayuda de una regla milimetrada se definió la longitud de trabajo (la cual es un milímetro menos que la longitud real de diente) y se registró en una tabla de recolección de datos. La longitud de trabajo de todas las muestras se estandarizó entre 19 y 20 mm y los dientes con mayor longitud se desgastaron en oclusal hasta obtener una longitud dentro del rango establecido.

Dispositivo para recolectar los residuos de la extrusión apical: Se basó en el modelo de Myers \& Montgomery (1991) (Fig. 1). Todos los recipientes se recubrieron con goma dique con el fin de prevenir que el operador vea la extrusión apical durante la fase de tratamiento. Antes de iniciado el procedimiento de instrumentación los tubos se pesaron en una balanza de precisión analítica (Modelo, AUX120 Shimatzu, Japón). Se realizaron tres mediciones consecutivas para cada tubo y el valor promedio se registró.

Preparación Químico Mecánica: La instrumentación se realizó según las indicaciones descrita por el fabricante por un solo operador especialista en endodoncia, utilizando el motor endodóntico Reciproc (VDW) programado según el instrumento utilizado, controlando tiempo y cinemática (Bürklein \& Schäfer). Se utilizó para todos los grupos como solución irrigante (2 $\mathrm{ml}$ ) hipoclorito de sodio al $5 \%$ en jeringas monoject (KV supply) con aguja de ranura lateral, entre cada lima. La penetración de la aguja fue a un límite de $3 \mathrm{~mm}$ de la longitud de trabajo y controlada con topes de silicona. La patencia apical fue constatada con una lima $\mathrm{K} \mathrm{N}^{\circ} 15$ entre cada instrumento. 
Los dientes fueron divididos aleatoriamente en cuatro grupos de 12 dientes cada uno:

Grupo 1 - Rotatorio / Irrigación pasiva: Se usó el motor endodóntico Reciproc (VDW) programado para sistema de limas Mtwo, con un movimiento rotatorio convencional con torque sugerido por el fabricante entre 300 y 350 rpm, alcanzado la longitud de trabajo del conducto radicular con todos los instrumentos de la siguiente manera: (1) Mtwo 10/.04, (2) Mtwo 15/.05, (3) Mtwo 20/.06, (4) Mtwo 25/.06, (5) Mtwo 30/.04, (6) Mtwo 35/.04 y (7) Mtwo 40/.04.

Grupo 2 - Reciprocante / Irrigación pasiva: Se usó el motor endodóntico Reciproc (VDW), con un movimiento reciproco, programado para sistema de limas Reciproc utilizadas en el siguiente orden: R25 25/.08 y R40 40/.06. Los instrumentos se utilizaron con un movimiento de picoteo sin presión, aproximadamente por 1-2 segundos avanzando $2 \mathrm{~mm}$ cada vez, hasta llegar a longitud de trabajo.

Grupo 3 - Rotatorio / Irrigación Activa: Se realizó la instrumentación como en la descripción del grupo 1 , se irrigó con $2 \mathrm{ml}$ de hipoclorito de sodio al $5 \%$ activándolo con el uso de Endoactivator (Dentsply Maillefer, Ballaigues, Suiza), su punta activada se ubicó a $3 \mathrm{~mm}$ de la longitud de trabajo durante 1 minuto.

Grupo 4 - Reciprocante - Irrigación Activa: Se realizó la instrumentación como en la descripción del grupo 2, luego esta se complementó con irrigación activa (Endoactivator), de la misma forma que en la descripción del grupo 3.

Como irrigación final en todos los grupos se utilizaron $2 \mathrm{ml}$ de EDTA $17 \%$ por un minuto, luego irrigación con $2 \mathrm{ml}$ de hipoclorito de sodio al $5 \%$ y secado con conos de papel.

Cuantificación del material sólido y líquido extruido por el foramen apical: La cuantificación del material extruido fue realizada por otro operador que no conocía la asignación de grupos. los dispositivos fueron desmontados y los tubos Eppendorf fueron retirados. El material extruido adherido a la superficie externa radicular del diente se raspó con una cureta periodontal y recolectado dentro del tubo.

Los tubos Eppendorf fueron centrifugados durante 5 minutos a una velocidad de $13200 \mathrm{rpm}$, luego el irrigante fue cuidadosamente retirado y cuantificado por medio de jeringas de insulina, quedando solo el material sólido en el tubo (Kus, tarci et al.), e inmediatamente fueron incubados a temperatura de 37,5 ${ }^{\circ} \mathrm{C}$, durante dos horas para lograr el completo secado.

Para el análisis de todas las variables se utilizó el programa SPSSv 17.0 (SPSS Inc., Chicago, IL, USA), inicialmente se realizó un contraste de normalidad con el test Shapiro Wilk y luego a través un análisis de varianza ANOVA.

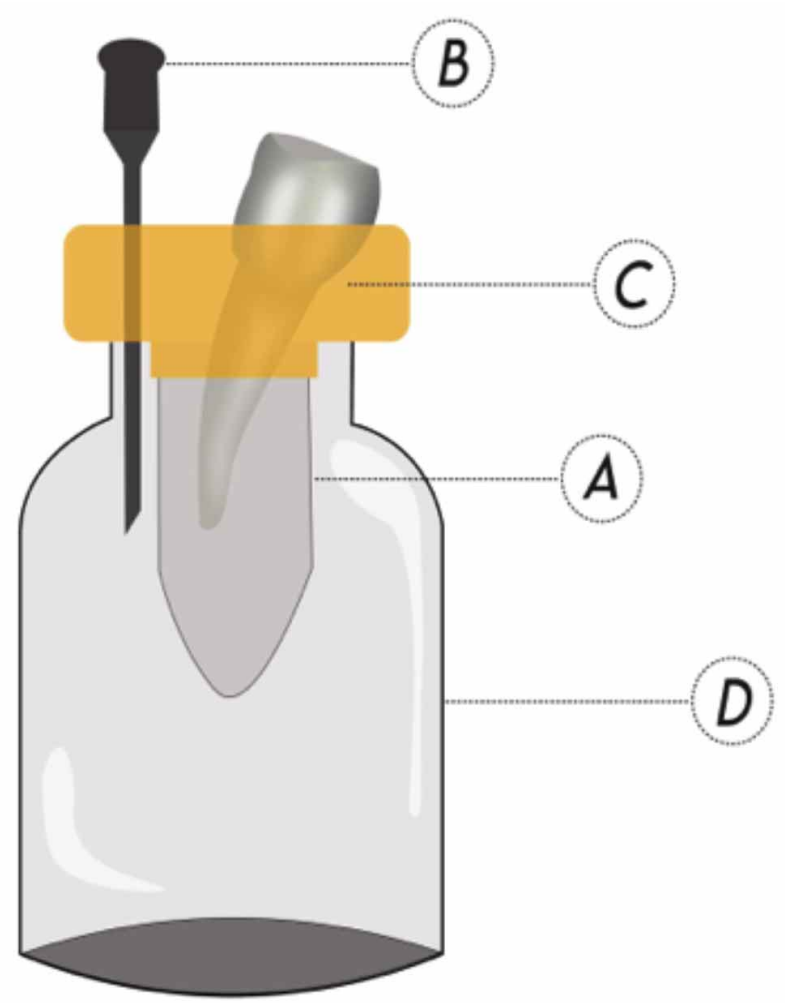

Fig. 1. Representación esquemática del dispositivo preparado para la evaluación de la extrusión apical (A) Tubo eppendorf en el cual la extrusión apical es recolectada. (B) Aguja insertada en el tapón de goma para equilibrar las presiones interna y externa. (C) Diente asegurado al tapón de goma. (D) Recipiente contenedor.

\section{RESULTADOS}

En todos los grupos se produjo extrusión apical de barro dentinario. El mayor valor se observó en el grupo Reciprocante - Irrigación activa (0,001084 + $0,001492 \mathrm{~g})$ y el menor valor en el grupo Rotatorio irrigación pasiva $(0,000341+0,000347 \mathrm{~g})$. No se identificaron diferencias estadísticamente significativas entre los grupos $(P=0,068)$. 
SIERRA-CRISTANCHO, A.; GÓMEZ, V. D.; GAJARDO, M. F. \& CORREA, S. V. Extrusión apical de barro dentinario e irrigante producidos por dos sistemas de instrumentación de níquel titanio al utilizar irrigación pasiva o activa. Int. J. Odontostomat., 13(1):51-57, 2019.

Tabla I. Cantidad de extrusión apical de barro dentinario (gramos) e irrigante (mililitros) después de la preparación químicomecánica.

\begin{tabular}{|c|c|c|c|c|c|c|c|c|c|}
\hline \multirow[b]{2}{*}{ İupos } & \multicolumn{5}{|c|}{ Extrusión barro dentinario } & \multicolumn{4}{|c|}{ Extrusión irrigante } \\
\hline & $\mathrm{N}$ & Media & Desv. Est. & Mínimo & Máximo & Media & Desv.Est. & Mínimo & Máximc \\
\hline Mtwo - Pasiva & 12 & 0,000341 & 0,000347 & 0 & 0,00114 & $0,01167^{*}$ & 0,02725 & 0 & 0,07 \\
\hline ․ Reciproc - Pasiva & 12 & 0,000788 & 0,0008 & 0 & 0,0027 & 0,0175 & 0,02379 & 0 & 0,06 \\
\hline 3. Mtwo - Activa & 12 & 0,00086 & 0,000748 & 0,00017 & 0,00224 & 0,05 & 0,0671 & 0 & $0,1 \mathrm{~s}$ \\
\hline 1. Reciproc - Activa & 12 & 0,001084 & 0,001492 & 0 & 0,00367 & $0,1125^{*}$ & 0,1682 & 0 & 0,47 \\
\hline
\end{tabular}

* Indica grupos significativamente diferentes $(p<0,05)$.

La extrusión apical de irrigante también se produjo en todos los grupos del estudio. El grupo Rotatorio - irrigación pasiva $(0,01167+0,02725 \mathrm{ml})$ presentó la menor extrusión de irrigante y la mayor el grupo Reciprocante -Irrigación activa $(0,1125+$ $0,1682 \mathrm{ml}$ ), en este aspecto se determinó que entre ambos existen diferencias estadísticamente significativas $(P<0,05)$, entre los demás grupos experimentales no se detectaron diferencia estadísticas $(P>0,05)$. Las medias y las desviaciones estándar para todos los grupos se muestran en la Tabla I.

\section{DISCUSIÓN}

El propósito de este estudio fue comparar la cantidad de extrusión apical de barro dentinario e irrigante, producida durante la preparación químico-mecánica, por dos sistemas de instrumentación mecanizada, utilizando irrigación pasiva o activa.

En este estudio se evaluaron dos tipos de instrumento de níquel titanio, el sistema Mtwo con 7 limas, de movimiento rotatorio continuo en sentido de las manecillas del reloj y el sistema reciprocante Reciproc con 2 limas, de movimiento reciproco, con ángulos de rotación hacia la izquierda y hacia la derecha de $150^{\circ}$ y $30^{\circ}$. Estos sistemas presentan características similares en el diseño de la sección transversal del instrumento, una forma de $\mathrm{S}$ itálica, de poca masa, con márgenes de corte afilados, lo que les confiere una eficacia de corte y porcentaje de extrusión similar (Bürklein \& Schäfer; Caviedes-Bucheli et al., 2018). Estudios previos concluyeron que el diseño del instrumento y el tipo de movimiento, presentan más relevancia que la cantidad de instrumentos utilizados, al evaluar el grado de extrusión apical entre sistemas de movimiento recíproco y rotatorio (Tinoco et al., 2014; Xavier et al., 2015; Silva et al., 2016a,b; Uzun et al., 2016).
Los resultados revelan que en todos los grupos experimentales se observó extrusión de barro dentinario, no presentando diferencias estadísticamente significativas. En relación a la extrusión de irrigantes, puede afirmarse que el mayor índice se evidenció en el sistema Reciproc irrigación activa y el menor sistema Mtwo - irrigación pasiva. Se determinó que existen diferencias estadísticamente significativas entre ambos grupos, lo que indica que ninguno de los sistemas evaluados de instrumentación e irrigación evita la extrusión apical. También, se pudo establecer que se produce una mayor extrusión apical de irrigantes cuando se utiliza irrigación activa (Endoactivator), lo que concuerda con lo reportado por investigaciones anteriores, que afirman que los sistemas complementarios de la irrigación como: ultrasonido, Endovac o láser, presentan una mayor extrusión de irrigantes, al compararlos con técnicas manuales tradicionales (Alkahtani et al.; Gupta et al.; Arslan et al.; Helvacıoglu Kıvanç et al.).

Independiente de la técnica de irrigación, el sistema reciprocante Reciproc presenta mayor extrusión que el rotatorio Mtwo, esto concuerda con estudios previos que relacionan los sistemas de movimiento reciprocante con una mayor extrusión apical (Nayak et al.; Tinoco et al.; Lu et al.; Silva et al., 2016a,b; Uzun et al.). Basado en estos resultados, es importante indicar que el instrumento reciprocante podría producir más barro dentinario al contacto con el canal radicular, debido a que su masa es mayor que el de una lima tradicional, con su diseño de lima única reemplazaría el uso de 3 o 4 limas con solo un instrumento, además, el movimiento reciprocante en sí podría señalarse como el responsable de la mayor extrusión apical. Cabe anotar que lo anteriormente reportado es imposible afirmarlo de manera absoluta debido a las limitantes de este estudio, por lo tanto se recomienda realizar estudios adicionales bajo condiciones controladas para evaluar esta hipótesis. 
Muchos factores pueden influir al realizar modelos experimentales donde se pretende determinar la extrusión apical producida durante la PQM. Estos factores van desde el tipo de diente, el tamaño y curvatura del conducto, la técnica de instrumentación (Ferraz et al.), el tipo y tamaño del instrumento, la determinación de la longitud de trabajo, diámetro apical (Teixeira et al., 2015), tipo y cantidad de la solución irrigante (Parirokh et al.; Silva et al., 2016a,b) hasta el sistema de recolección del material extruido. En el presente estudio, para minimizar los efectos de los factores antes mencionados, se crearon las condiciones estándar para todos los grupos (distintas del sistema de instrumentación utilizado). Se seleccionaron premolares mandibulares, de conducto radicular único, con una raíz recta y ápices maduros (por lo que los resultados observados no se deben generalizar a los dientes condesarrollo radicular inmaduro y ápices abiertos), se determinó la longitud de trabajo a $1 \mathrm{~mm}$ del foramen apical con una permeabilidad apical dada por una lima 15/02. La instrumentación fue realizada por un solo operador, para darle una mayor objetividad al proceso, con una presión, fuerza y forma de manipular los instrumentos endodónticos e irrigantes similar en todos los canales radiculares tratados.

La metodología utilizada se basó en la propuesta por Myers \& Montgomery, que utiliza como irrigante agua destilada. En este estudio se propuso que el diseño experimental fuera lo más cercano a la realidad clínica actual, usando hipoclorito de sodio al $5 \%$ como irrigante principal, acompañado de un lavado final con EDTA al $17 \%$ e hipoclorito de sodio al $5 \%$. Un inconveniente al incubar el hipoclorito de sodio a $37,5^{\circ} \mathrm{C}$ es la formación de cristales dentro del tubo Eppendorf las cuales alteran el resultado final del peso obtenido, al mezclarse con el detritus extruido. Para aumentar la eficacia del método, se centrifugaron a $12.300 \mathrm{rpm}$ durante 5 minutos los tubos Eppendorf, y se obtuvo un precipitado de la fase sólida y un sobrenadante de la fase líquida. Además, con el fin de eliminar el efecto de la cantidad de irrigante en la extrusión de desechos, se mantuvo constante la cantidad utilizada.

Un cierto grado de precaución se debe tomar cuando se transfieren estos resultados a la situación clínica. El método in vitro tenía el ápice suspendido en el aire, mientras que in vivo el ápice está rodeado de tejidos periapicales, que podrían ayudar a limitar la extrusión apical en cierta medida (Lu et al.; Nevares et al.) El inconveniente de esta configuración experimental es la ausencia de una contrapresión física proporcionada por los tejidos periapicales, se dice que serían capaces de resistir la extrusión de sólidos y líquidos. En el presente estudio, no se intentó simular la resistencia periapical.

La extrusión de barro dentinario y las soluciones de irrigación han sido señalados como los responsables del dolor post operatorio y reagudizaciones (Violich \& Chandler; Onay et al.). Estudios clínicos donde se han evaluado marcadores biológicos de la expresión de neuropéptidos como la sustancia P (SP) y Polipéptido relacionado con el gen de la calcitonina (CGRP) como consecuencia del uso de sistemas de instrumentación mecanizada, han asociado la extrusión apical como unos de los mayores agentes etiológicos de la Periodontitis Apical Sintomática post instrumentación y concluyen que todos los sistemas rotatorios generan la liberación de neuropéptidos en el ligamento periodontal (Caviedes-Bucheli et al., 2016). Sin embargo, es necesario llevar a cabo más estudios in vitro y estudios clínicos que cuantifiquen otros mediadores inflamatorios con nuevas metodologías que permitan asociar la extrusión apical con procesos inflamatorios en el ligamento periodontal como la causa de Periodontitis Apical Sintomática.

\section{CONCLUSIONES}

Bajo las condiciones de este estudio, todas las técnicas de instrumentación evaluadas producen extrusión apical de barro dentinario e irrigantes. El sistema de instrumentación reciprocante fue asociado a una mayor extrusión de irrigante al ser comparada con el uso del sistema rotatorio, independiente del tipo de irrigación utilizada. El diseño y tipo de movimiento del instrumento (rotatorio o reciprocante) podrían influenciar la extrusión apical de barro dentinario y/o irrigantes, por lo cual deberían ser evaluados en futuros estudios clínicos.

\section{AGRADECIMIENTOS}

Ignacio Fuentevilla, Candidato a Doctor del Programa de Biociencias Moleculares de la Universidad Andrés Bello. Por el análisis estadístico de los datos y su apoyo logístico en el Laboratorio de Microbiología y Biotecnología Oral para realizar la fase experimental del trabajo. Javiera Herrera Alvear, Diseñadora gráfica por el desarrollo de la figura. 
SIERRA-CRISTANCHO, A.; GÓMEZ, D. V.; GAJARDO, F. M. \& CORREA, V. S. Apical extrusion of dentin and irrigant smear layer produced by two nickel titanium instrumentation systems when using passive or active irrigation. Int. J. Odontostomat., 13(1):55-57, 2019.

ABSTRACT: The smear layer produced during endodontic treatment can be extruded into the periradicular tissue together with the irrigant, producing inflammation and postoperative pain. The purpose of this in vitro study was to compare the amount of apical extrusion, produced during the endodontic preparations with two rotary instrumentation systems using passive and active irrigation. Forty-eight (48) mandibular premolars were randomly assigned to 4 groups ( $n=12$ teeth), (1) Rotatory - passive, (2) Reciprocating passive, (3) Rotatory-active, (4) Reciprocating-active. The root canals were instrumented according to the manufacturer's instructions and irrigated with $5 \%$ sodium hypochlorite. Extruded debris and irrigant were collected in previously weighed Eppendorf tubes. The extrusion values were calculated for each group. The data were analyzed with the ANOVA analysis of variance. Results: Apical extrusion was produced in all groups. No statistically significant differences were found in the apical extrusion of smear layer, between the groups studied $(P=0.068)$, however, when analyzing the extrusion of irrigants, a statistically significant difference was evidenced between groups 1 and $4(P<0.05)$., among the other groups no statistically significant differences were observed. $(P>0.05)$. Under the conditions of this study, the reciprocating and rotary systems, extrude solid and liquid, being the reciprocating system the one that produces more extrusion of liquid, with both active and passive irrigation.

KEY WORDS: apical extrusion, smear layer, mechanized instrumentation.

\section{REFERENCIAS BIBLIOGRÁFICAS}

Aksel, H.; Askerbeyli, S.; Canbazoglu, C. \& Serper, A. Effect of needle insertion depth and apical diameter on irrigant extrusion in simulated immature permanent teeth. Braz. Oral Res., 28:1-6, 2014.

Alkahtani, A.; Al Khudhairi, T. D. \& Anil, S. A. A comparative study of the debridement efficacy and apical extrusion of dynamic and passive root canal irrigation systems. BMC Oral Health, 14:12, 2014.

American Association of Endodontists (AAE). Glossary of Endodontic Terms. $9^{\text {th }}$ ed. Chicago, American Association of Endodontists, 2015.

Arslan, H.; Akcay, M.; Ertas, H.; Capar, I. D.; Saygili, G. \& Mese, M. Effect of PIPS technique at different power settings on irrigating solution extrusion. Lasers Med. Sci., 30(6):1641-5, 2015.

Bürklein, S. \& Schäfer, E. Apically extruded debris with reciprocating single-file and full-sequence rotary instrumentation systems. J. Endod., 38(6):850-2, 2012.
Caviedes-Bucheli, J.; Castellanos, F.; Vasquez, N.; Ulate, E. \& Munoz, $H$. R. The influence of two reciprocating single-file and two rotaryfile systems on the apical extrusion of debris and its biological relationship with symptomatic apical periodontitis. A systematic review and meta-analysis. Int. Endod. J., 49(3):255-70, 2016.

Caviedes-Bucheli, J.; Moreno, J. O.; Carreño, C. P.; Delgado, R.; Garcia, D. J.; Solano, J.; Diaz, E. \& Munoz, H. R. The effect of single-file reciprocating systems on Substance $\mathrm{P}$ and Calcitonin gene-related peptide expression in human periodontal ligament. Int. Endod. J., 46(5):419-26, 2013.

Caviedes-Bucheli, J.; Rios-Osorio, N.; Rey-Rojas, M.; LagunaRivero, F.; Azuero-Holguin, M. M.; Diaz, L. E.; Curtidor, H.; Castaneda-Ramirez, J. J. \& Munoz, H. R. Substance P and Calcitonin gene-related peptide expression in human periodontal ligament after root canal preparation with Reciproc Blue, WaveOne Gold, XP EndoShaper and hand files. Int. Endod. J., 51(12):1358-66, 2018.

Chang, J. W.; Cheung, A. W. \& Cheung, G. S. Effect of root canal dimensions, injection rate, and needle design on the apical extrusion of an irrigant: an in vitro study. J. Investig. Clin. Dent., 6(3):221-7, 2015.

Ferraz, C. C.; Gomes, N. V.; Gomes, B. P.; Zaia, A. A.; Teixeira, F. B. \& Souza-Filho, F. J. Apical extrusion of debris and irrigants using two hand and three engine-driven instrumentation techniques. Int. Endod. J., 34(5):354-8, 2001.

Gupta, J.; Nikhil, V. \& Jha, P. Corelation between machines assisted endodontic irrigant agitation and apical extrusion of debris and irrigant: a laboratory study. Scientific World Journal, 2014:346184, 2014.

Helvacıoglu Kıvanç, B.; Deniz Arısu, H.; Yanar, N. Ö.; Silah, H. M.; Inam, R. \& Görgül, G. Apical extrusion of sodium hypochlorite activated with two laser systems and ultrasonics: a spectrophotometric analysis. BMC Oral Health, 15:71, 2015.

Huiz Peeters, H.; Suardita, K.; Mooduto, L. \& Gutknecht, N. Extrusion of irrigant in open apex teeth with periapical lesions following laser-activated irrigation and passive ultrasonic irrigation. Iran Endod. J., 13(2):169-75, 2018.

Kustarci, A.; Akpinar, K. E. \& Er, K. Apical extrusion of intracanal debris and irrigant following use of various instrumentation techniques. Oral Surg. Oral Med. Oral Pathol. Oral Radiol. Endod., 105(2):257-62, 2008.

Lu, Y.; Chen, M.; Qiao, F. \& Wu, L. Comparison of apical and coronal extrusions using reciprocating and rotary instrumentation systems. BMC Oral Health, 15:92, 2015.

Myers, G. L. \& Montgomery, S. A comparison of weights of debris extruded apically by conventional filing and Canal Master techniques. J. Endod., 17(6):275-9, 1991.

Nayak, G.; Singh, I.; Shetty, S. \& Dahiya, S. Evaluation of apical extrusion of debris and irrigant using two new reciprocating and one continuous rotation single file systems. J. Dent. (Tehran), 11(3):302-9, 2014.

Nevares, G.; Xavier, F.; Gominho, L.; Cavalcanti, F.; Cassimiro, M.; Romeiro, K.; Alvares, P.; Queiroz, G.; Sobral, A. P.; Gerbi, M.; Silveira, M. \& Albuquerque, D. Apical extrusion of debris produced during continuous rotating and reciprocating motion. Scientific World Journal, 2015:267264, 2015.

Onay, E. O.; Ungor, M. \& Yazici, A. C. The evaluation of endodontic flare-ups and their relationship to various risk factors. BMC Oral Health, 15:142, 2015.

Parirokh, M.; Jalali, S.; Haghdoost, A. A. \& Abbott, P. V. Comparison of the effect of various irrigants on apically extruded debris after root canal preparation. J. Endod., 38(2):196-9, 2012.

Rodríguez-Figueroa, C.; McClanahan, S. B. \& Bowles, W. R. Spectrophotometric determination of irrigant extrusion using passive ultrasonic irrigation, EndoActivator, or syringe irrigation. J. Endod., 40(10):1622-6, 2014. 
SIERRA-CRISTANCHO, A.; GÓMEZ, V. D.; GAJARDO, M. F. \& CORREA, S. V. Extrusión apical de barro dentinario e irrigante producidos por dos sistemas de instrumentación de níquel titanio al utilizar irrigación pasiva o activa. Int. J. Odontostomat., 13(1):51-57, 2019.

Silva, E. J.; Carapiá, M. F.; Lopes, R. M.; Belladonna, F. G.; Senna, P. M.; Souza, E. M. \& De-Deus, G. Comparison of apically extruded debris after large apical preparations by full-sequence rotary and single-file reciprocating systems. Int. Endod. J., 49(7):700-5, 2016a.

Silva, P. B.; Krolow, A. M.; Pilownic, K. J.; Casarin, R. P.; Lima, R. K.; Leonardo, R. de T. \& Pappen, F. G. Apical extrusion of debris and irrigants using different irrigation needles. Braz. Dent. J., 27(2):192-5, 2016b.

Sipaviciute', E. \& Maneliene', R. Pain and flare-up after endodontic treatment procedures. Stomatologija, 16(1):25-30, 2014.

Sowmya, H. K.; Subhash, T. S.; Goel, B. R.; Nandini, T. N. \& Bhandi, S. H. Quantitative assessment of apical debris extrusion and intracanal debris in the apical third, using hand instrumentation and three rotary instrumentation systems. J. Clin. Diagn. Res., 8(2):206-10, 2014.

Teixeira, J. M.; Cunha, F. M.; Jesus, R. O.; Silva, E. J.; Fidel, S. R. \& Sassone, L. M. Influence of working length and apical preparation size on apical bacterial extrusion during reciprocating instrumentation. Int. Endod. J., 48(7):648-53, 2015.

Tinoco, J. M.; De-Deus, G.; Tinoco, E. M.; Saavedra, F.; Fidel, R. A. \& Sassone, L. M. Apical extrusion of bacteria when using reciprocating single-file and rotary multifile instrumentation systems. Int. Endod. J., 47(6):560-6, 2014.

Uzun, I.; Güler, B.; Özyürek, T. \& Tunc, T. Apical extrusion of debris using reciprocating files and rotary instrumentation systems. Niger. J. Clin. Pract., 19(1):71-5, 2016.

Violich, D. R. \& Chandler, N. P. The smear layer in endodontics - a review. Int. Endod. J., 43(1):2-15, 2010.

Xavier, F.; Nevares, G.; Romeiro, M. K.; Gonçalves, K.; Gominho, L. \& Albuquerque, D. Apical extrusion of debris from root canals using reciprocating files associated with two irrigation systems. Int. Endod. J., 48(7):661-5, 2015.

Yost, R. A.; Bergeron, B. E.; Kirkpatrick, T. C.; Roberts, M. D.; Roberts, H. W.; Himel, V. T. \& Sabey, K. A. Evaluation of 4 different irrigating systems for apical extrusion of sodium hypochlorite. J. Endod., 41(9):1530-4, 2015.

\author{
Dirección para correspondencia: \\ Alfredo Sierra-Cristancho \\ Santa Rosa 17 Oficina 72 \\ Santiago \\ CHILE
}

Email: dr_alfredosierra@hotmail.com

Recibido : 18-08-2018

Aceptado: 19-10-2018 